\title{
Stereoselective Syntheses of Functionalized Precursors of the CDEF and CDE 2,6-Dideoxy-Tetra- and Trisaccharide Units of Durhamycins A and B
}

\section{Supporting Information}

\author{
Timothy B. Durham and William R. Roush
}

General Methods: All reaction solvents were purified before use. Tetrahydrofuran, dichloromethane, and toluene were purified by passage through a solvent column ${ }^{1}$ composed of activated A-1 alumina. Unless otherwise indicated, all commercial reagents were used as received without further purification.

Physical Properties and Spectroscopic Measurements: Proton nuclear magnetic resonance $\left({ }^{1} \mathrm{H}\right.$ NMR) spectra and carbon-13 $\left({ }^{13} \mathrm{C}\right)$ NMR spectra were recorded on a Varian VXR-400 spectrometer at $400 \mathrm{MHz}$ and $100 \mathrm{MHz}$ respectively, or on a Varian Inova-500 spectrometer at $500 \mathrm{MHz}$ and 125 respectively. The proton signal of residual, non-deuterated solvent $\left(\delta 7.26 \mathrm{ppm}\right.$ for $\mathrm{CHCl}_{3} ; \delta 7.16 \mathrm{ppm}$ for $\left.\mathrm{C}_{6} \mathrm{D}_{5} \mathrm{H}\right)$ or tetramethylsilane $\left(\delta 0 \mathrm{ppm}, \mathrm{CDCl}_{3}\right)$ was used as an internal reference for ${ }^{1} \mathrm{H}$ spectra. For ${ }^{13} \mathrm{C}$ spectra, chemical shifts are reported relative to the $\delta 77.23 \mathrm{ppm}$ resonance of $\mathrm{CDCl}_{3}$ or the $\delta 128.39 \mathrm{ppm}$ resonance of $\mathrm{C}_{6} \mathrm{D}_{6}$. Coupling constants are reported in Hz. Infrared (IR) spectra were recorded as thin films on a Perkin-Elmer Spectrum 1000 FTIR. Optical rotations were measured on a Rudolph Autopol III polarimeter using a quartz cell with $1 \mathrm{~mL}$ capacity and a $10 \mathrm{~cm}$ path length. Mass spectra were recorded on a VG 70-250-S spectrometer manufactured by Micromass Corp. (Manchester UK).

Analytical thin layer chromatography (TLC) was performed on Kieselgel $60 \mathrm{~F}_{254}$ glass plates precoated with a $0.25 \mathrm{~mm}$ thickness of silica gel. The TLC plates were visualized with UV light and/or by staining with Hanessian stain (a mixture of ceric sulfate and ammonium molybdate in aqueous sulfuric acid), $\mathrm{KMnO}_{4}$, or $p$-anisaldehyde solutions. Column chromatography was generally performed using Kieselgel 60 (230-400 mesh) silica gel. Unless noted otherwise, all compounds isolated by chromatography were sufficiently pure by ${ }^{1} \mathrm{H}$ NMR analysis for use in subsequent reactions.

HPLC purifications were performed by using a HPLC system composed of two Rainin HPXL pumps connected to various Dynamax ${ }^{\circledR}$ axial compression columns packed with Rainin $60 \AA \AA$ irregular silica gel. Samples were loaded into the system with a $2 \mathrm{~mL}$ Rheodyne 7125 injector and were detected using either a Rainin Dynamax ${ }^{\circledR}$ UV-C detector or a Rainin Dynamax ${ }^{\circledR}$ RI-1 detector. Integration of the various signals was performed using the reprocessing program within the Dynamax ${ }^{\circledR}$ HPLC Method Manager.

Experimental:

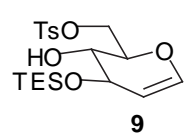

1. $\mathrm{NaBr}, \mathrm{NaHCO}_{3}$,
$\mathrm{DMF}, 65{ }^{\circ} \mathrm{C}$

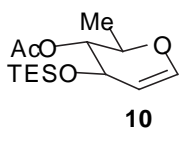

3. $\mathrm{Bu}_{3} \mathrm{SnH}, \mathrm{AIBN}, \mathrm{C}_{6} \mathrm{H}_{6}$,

4-Acetyl-6-bromo-3-(triethylsilyl)-D-glucal. Tosylate 9 (8.44 g, $20.3 \mathrm{mmol}){ }^{2} \mathrm{NaBr}(29.0 \mathrm{~g}, 282 \mathrm{mmol})$, and sodium bicarbonate $(4.4 \mathrm{~g}, 52 \mathrm{mmol})$ were suspended in dry DMF $(130 \mathrm{~mL})$ under a nitrogen atmosphere. The resulting mixture was heated to $65^{\circ} \mathrm{C}$ and stirred vigorously for $10 \mathrm{~h}$. The mixture was cooled to $23^{\circ} \mathrm{C}$ and diluted with EtOAc. The resulting solution was washed with water ( $\mathrm{x}$ 4) followed by saturated $\mathrm{NaCl}$ solution, dried over $\mathrm{Na}_{2} \mathrm{SO}_{4}$, filtered, and concentrated to give a clear oil $(6.3 \mathrm{~g}, 95 \%)$.

The bromide was then dissolved in dry $\mathrm{CH}_{2} \mathrm{Cl}_{2}(200 \mathrm{~mL})$ under a nitrogen atmosphere. The resulting solution was cooled to $0{ }^{\circ} \mathrm{C}$ and DMAP $(134 \mathrm{mg}, 1.09 \mathrm{mmol}), \mathrm{Et}_{3} \mathrm{~N}(4.2 \mathrm{~mL}, 30 \mathrm{mmol})$, and $\mathrm{Ac}_{2} \mathrm{O}(2.8 \mathrm{~mL}, 30 \mathrm{mmol})$ were 
successively added. After $30 \mathrm{~min}$, the reaction mixture was warmed to $23{ }^{\circ} \mathrm{C}$ and stirred for $10 \mathrm{~h}$. The mixture was diluted with EtOAc. The resulting solution was washed with water, saturated sodium bicarbonate solution, and saturated $\mathrm{NaCl}$ solution, dried over $\mathrm{Na}_{2} \mathrm{SO}_{4}$, filtered, and concentrated to give an oil which was purified by flash chromatography $\left(20: 1\right.$ hexanes/EtOAc) to give the title compound as a clear oil $(6.09 \mathrm{~g}, 82 \%):[\alpha]_{\mathrm{D}}{ }^{27^{\circ} \mathrm{C}}=-35.4^{\circ}(\mathrm{c}$ $\left.=1.0, \mathrm{CH}_{2} \mathrm{Cl}_{2}\right) ;{ }^{1} \mathrm{H} \mathrm{NMR}\left(500 \mathrm{MHz}, \mathrm{CDCl}_{3}\right) \delta 6.39(\mathrm{~d}, J=1.00,6.50 \mathrm{~Hz}, 1 \mathrm{H}), 5.11(\mathrm{td}, J=1.00,4.00 \mathrm{~Hz}, 1 \mathrm{H}), 4.81$ $(\mathrm{ddd}, J=1.00,4.00,6.50 \mathrm{~Hz}, 1 \mathrm{H}), 4.27$ (dddd, $J=1.00,5.00,5.00,8.00 \mathrm{~Hz}, 1 \mathrm{H}), 4.13(\mathrm{tt}, J=1.00,4.00,5.00 \mathrm{~Hz}$, $1 \mathrm{H}), 3.66(\mathrm{dd}, J=5.50,11.00 \mathrm{~Hz}, 1 \mathrm{H}), 3.62(\mathrm{dd}, J=8.00,11.00 \mathrm{~Hz}, 1 \mathrm{H}), 2.11(\mathrm{~s}, 3 \mathrm{H}), 0.96(\mathrm{t}, 8.00 \mathrm{~Hz}, 9 \mathrm{H}), 0.62$ $(\mathrm{q}, J=8.00 \mathrm{~Hz}, 6 \mathrm{H}){ }^{13} \mathrm{C} \mathrm{NMR}\left(125 \mathrm{MHz}, \mathrm{CDCl}_{3}\right) \delta 169.81,142.97,102.75,75.51,71.57,64.01,30.06,21.14,6.85$, 4.87; IR (thin film) $1743 \mathrm{~cm}^{-1}$; HRMS (ES) calcd for $\mathrm{C}_{14} \mathrm{H}_{25} \mathrm{O}_{4} \mathrm{BrSiNa}(\mathrm{M}+\mathrm{Na})^{+} 387.0603$, found $387.0600 \mathrm{~m} / \mathrm{z}$.

4-Acetyl-6-deoxy-3-(triethylsilyl)-D-glucal (10). Bromide 10 (1.02 g, 2.80 mmol) and AIBN (257 mg, 1.57 $\mathrm{mmol})$ were dissolved in dry benzene $(30 \mathrm{~mL})$ under a nitrogen atmosphere. To the resulting solution was added $\mathrm{Bu}_{3} \mathrm{SnH}(0.80 \mathrm{~mL}, 3.0 \mathrm{mmol})$ and the mixture was heated to reflux. After $5 \mathrm{~h}$, the reaction was cooled to $23{ }^{\circ} \mathrm{C}$ and was stirred vigorously with $20 \% \mathrm{KF}$ solution $(20 \mathrm{~mL})$ for $10 \mathrm{~min}$. The resulting mixture was transferred to a separatory funnel and diluted with EtOAc $(100 \mathrm{~mL})$. The aqueous layer was discarded and the organic phase was washed with $20 \% \mathrm{KF}$ solution (x 4), saturated $\mathrm{NaCl}$ solution, dried over $\mathrm{Na}_{2} \mathrm{SO}_{4}$, filtered, and concentrated to give an oil which was purified by flash chromatography (long column, $50: 1$ hexanes/EtOAc) to provide the title compound as a clear oil $(725 \mathrm{mg}, 90 \%):[\alpha]_{\mathrm{D}}{ }^{27^{\circ} \mathrm{C}}=-42.7^{\circ}\left(\mathrm{c}=0.3, \mathrm{CH}_{2} \mathrm{Cl}_{2}\right) ;{ }^{1} \mathrm{H} \mathrm{NMR}\left(500 \mathrm{MHz} \mathrm{CDCl}_{3}\right) \delta 6.31$ $(\mathrm{dd}, J=6.00,1.50 \mathrm{~Hz}, 1 \mathrm{H}), 4.92(\mathrm{dd}, J=6.00,8.00 \mathrm{~Hz}, 1 \mathrm{H}), 4.69(\mathrm{dd}, J=3.00,6.00 \mathrm{~Hz}, 1 \mathrm{H}), 4.55(\mathrm{~m}, 1 \mathrm{H}), 4.02$ $(\mathrm{m}, 1 \mathrm{H}), 2.10(\mathrm{~s}, 3 \mathrm{H}), 1.29(\mathrm{~d}, J=6.50 \mathrm{~Hz}, 1 \mathrm{H}), 0.96(\mathrm{t}, J=8.00 \mathrm{~Hz}, 9 \mathrm{H}), 0.60(\mathrm{~m}, 6 \mathrm{H}) ;{ }^{13} \mathrm{C} \mathrm{NMR}(125 \mathrm{MHz}$, $\left.\mathrm{CDCl}_{3}\right) \delta 170.10,143.77,103.50,75.23,72.86,66.77,21.27,16.92,6.91,5.02$; IR (thin film) $1750 \mathrm{~cm}^{-1}$; HRMS (ES) calcd for $\mathrm{C}_{14} \mathrm{H}_{26} \mathrm{O}_{4} \mathrm{SiNa}(\mathrm{M}+\mathrm{Na})^{+} 309.1498$, found $309.1509 \mathrm{~m} / \mathrm{z}$.
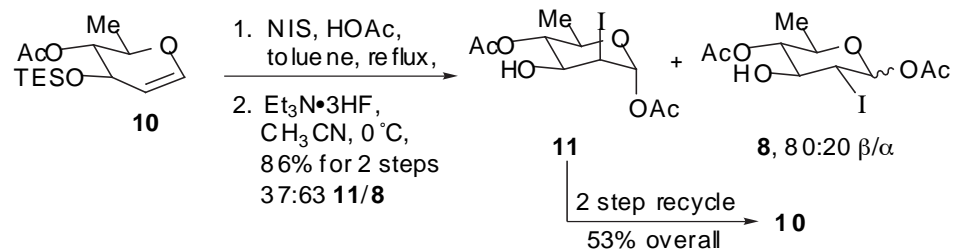

Acetyl 4- $O$-acetyl-6-deoxy-2-deoxy-2-iodo- $\alpha$-D-mannopyranoside (11) and $\alpha, \beta$-Acetyl 4- $O$-acetyl-6-deoxy2-deoxy-2-iodo-D-glucopyranoside (8). Galactal 10 (1.28 g, $4.46 \mathrm{mmol})$ was dissolved in dry toluene (50 $\mathrm{mL})$ under nitrogen in a round-bottom flask equipped with a reflux condensor. To the resulting solution was added $\mathrm{AcOH}$ $(1.00 \mathrm{~mL}, 17.5 \mathrm{mmol})$ followed by $N$-iodosuccinimide $(3.03 \mathrm{~g}, 13.5 \mathrm{mmol})$. The flask was then immediately plunged into a preheated $\left(>150^{\circ} \mathrm{C}\right)$ oil bath. The reaction mixture was allowed to reflux for 15 min and then removed from the bath and cooled to $23{ }^{\circ} \mathrm{C}$. The reaction was quenched by the addition of $10 \% \mathrm{Na}_{2} \mathrm{~S}_{2} \mathrm{O}_{3}$ and the resulting mixture was stirred vigorously until it had become colorless. The mixture was then diluted with EtOAc, transferred to a separatory funnel, and the aqueous layer was discarded. The organic phase was washed with $10 \% \mathrm{Na}_{2} \mathrm{~S}_{2} \mathrm{O}_{3}$ (x 3), saturated $\mathrm{NaCl}$ solution, dried over $\mathrm{Na}_{2} \mathrm{SO}_{4}$, filtered, and concentrated. The residue was purified by flash chromatography (4:1 hex/EtOAc) to provide the title compounds $(2.01 \mathrm{~g}, 95 \%)$ as yellow oil. The $39: 47: 14$ mixture of $\alpha$-manno/ $\beta$-gluco/ $\alpha$-gluco mixture of iodoacetate diastereomers was inseparable by flash chromatography and HPLC.

The mixture of carbohydrate derivatives $(1.72 \mathrm{~g}, 3.65 \mathrm{mmol})$ was dissolved in dry $\mathrm{CH}_{3} \mathrm{CN}(35 \mathrm{~mL})$ under a nitrogen atmosphere. The resulting solution was cooled to $0{ }^{\circ} \mathrm{C}$ and $\mathrm{Et}_{3} \mathrm{~N} \bullet 3 \mathrm{HF}(1.4 \mathrm{~mL}, 8.6 \mathrm{mmol})$ was added dropwise. The reaction was stirred for $5.5 \mathrm{~h}$, then warmed to $23{ }^{\circ} \mathrm{C}$ and diluted with EtOAc $(150 \mathrm{~mL})$. The solution was washed with saturated sodium bicarbonate solution (x 3), brine, dried over $\mathrm{Na}_{2} \mathrm{SO}_{4}$, filtered, and concentrated. The residue was purified by flash chromatography $(7: 1$ then $4: 1$ hex/EtOAc, glucoside 8 elutes first) to provide glucoside $8(720 \mathrm{mg}, 55 \%, 80: 20 \beta / \alpha)$ and mannoside $11(464 \mathrm{mg}, 36 \%)$ as white solids. The mixture of glucoside anomers was then recystallized from diethyl ether/hexanes to give $606 \mathrm{mg}$ of a white crystalline solid $(88: 12 \beta / \alpha$, if the recrystallization is stopped earlier, the ratio can be increased up to $98: 2$ ): Data for mannoside 11: $[\alpha]_{\mathrm{D}}{ }^{2 \mathrm{C}^{\circ} \mathrm{C}}=-$ $29.5^{\circ}\left(\mathrm{c}=0.2, \mathrm{CH}_{2} \mathrm{Cl}_{2}\right) ; \mathrm{mp}=124^{\circ} \mathrm{C}(\mathrm{d}) ;{ }^{1} \mathrm{H} \mathrm{NMR}\left(500 \mathrm{MHz}, \mathrm{CDCl}_{3}\right) \delta 6.35($ as, $1 \mathrm{H}), 4,93($ at $, J=9.50 \mathrm{~Hz}, 1 \mathrm{H})$, $4.49(\mathrm{dd}, J=1.50,4.00 \mathrm{~Hz}, 1 \mathrm{H}), 4.00(\mathrm{dddd}, J=6.50,6.50,6.50,9.50 \mathrm{~Hz}), 3.22(\mathrm{td}, J=4.00,9.00 \mathrm{~Hz}, 1 \mathrm{H}), 2.80(\mathrm{~d}$, $J=8.50 \mathrm{~Hz}, 1 \mathrm{H}), 2.15(\mathrm{~s}, 3 \mathrm{H}), 2.14(\mathrm{~s}, 3 \mathrm{H}), 1.25(\mathrm{~d}, J=6.50 \mathrm{~Hz}, 1 \mathrm{H}) ;{ }^{13} \mathrm{C} \mathrm{NMR}\left(125 \mathrm{MHz}, \mathrm{CDCl}_{3}\right) \delta 171.34$, 168.68, 94.85, 76.09, 69.53, 67.73, 36.43, 21.03, 20.98, 17.63; IR (thin film) 3474 (b), $1745 \mathrm{~cm}^{-1}$; HRMS (ES) calcd for $\mathrm{C}_{10} \mathrm{H}_{15} \mathrm{O}_{6} \mathrm{INa}(\mathrm{M}+\mathrm{Na})^{+} 380.9811$, found $380.9813 \mathrm{~m} / \mathrm{z}$.

Data for $\beta-8$ : $[\alpha]_{\mathrm{D}}^{27^{\circ} \mathrm{C}}=-11.7^{\circ}\left(\mathrm{c}=1.5, \mathrm{CH}_{2} \mathrm{Cl}_{2}\right) ; \mathrm{mp}=124^{\circ} \mathrm{C}(\mathrm{d}) ; \mathrm{mp}=148-150^{\circ} \mathrm{C}(\mathrm{d}) ;{ }^{1} \mathrm{H} \mathrm{NMR}(500 \mathrm{MHz}$, $\left.\mathrm{CDCl}_{3}\right) \delta 5.83(\mathrm{~d}, J=9.50 \mathrm{~Hz}, 1 \mathrm{H}), 4.67(\mathrm{dd}, J=9.00,9.50 \mathrm{~Hz}, 1 \mathrm{H}), 3.93(\mathrm{dd}, J=9.25,10.75,1 \mathrm{H}), 3.86(\mathrm{ddd}, J=$

$$
\mathrm{SI}-2
$$


4.00, 9.00,10.50,1H), $2.65(\mathrm{~m}, 1 \mathrm{H}), 2.17(\mathrm{~s}, 3 \mathrm{H}), 2.14(\mathrm{~s}, 3 \mathrm{H}), 1.25(\mathrm{~d}, J=6.50 \mathrm{~Hz}, 3 \mathrm{H}) ;{ }^{13} \mathrm{C} \mathrm{NMR}(100 \mathrm{MHz}$, $\left.\mathrm{CDCl}_{3}\right) \delta 170.94,169.11,93.86,76.44,75.52,71.40,33.60,21.12,21.00,17.47$; IR (thin film) $3480,1749,1731 \mathrm{~cm}^{-}$ ${ }^{1}$; HRMS (ES) calcd for $\mathrm{C}_{10} \mathrm{H}_{15} \mathrm{O}_{6} \mathrm{INa}(\mathrm{M}+\mathrm{Na})^{+} 380.9811$, found $380.9816 \mathrm{~m} / \mathrm{z}$.

Conversion of mannoside 11 to glucal derivative 10. Mannoside 11 (101 $\mathrm{mg}, 0.282 \mathrm{mmol}$ ) and imidazole (44 $\mathrm{mg}, 0.64 \mathrm{mmol})$ were dissolved in dry DMF $(1 \mathrm{~mL})$ under a nitrogen atmosphere. To the resulting solution was added TESCl $(57 \mu \mathrm{L}, 0.34 \mathrm{mmol})$. The mixture was stirred overnight and then diluted with EtOAc. The solution was washed with saturated sodium bicarbonate solution (x 2), brine, dried over $\mathrm{Na}_{2} \mathrm{SO}_{4}$, filtered, and concentrated. The residue was dried under vacuum and then dissolved in dry THF under a nitrogen atmosphere. The flask was covered with foil and LiI (366 mg, $2.74 \mathrm{mmol})$ was added. Upon complete consumption of the starting material (20 h), the mixture was diluted with EtOAc. The solution was washed with $10 \% \mathrm{Na}_{2} \mathrm{~S}_{2} \mathrm{O}_{4}$ solution (until colorless), brine, dried over $\mathrm{Na}_{2} \mathrm{SO}_{4}$, filtered, and concentrated. The residue was purified by flash chromatography (7: 1 hexanes/EtOAc) to give glucal derivative $\mathbf{1 0}(43 \mathrm{mg}, 53 \%)$ as a clear oil.

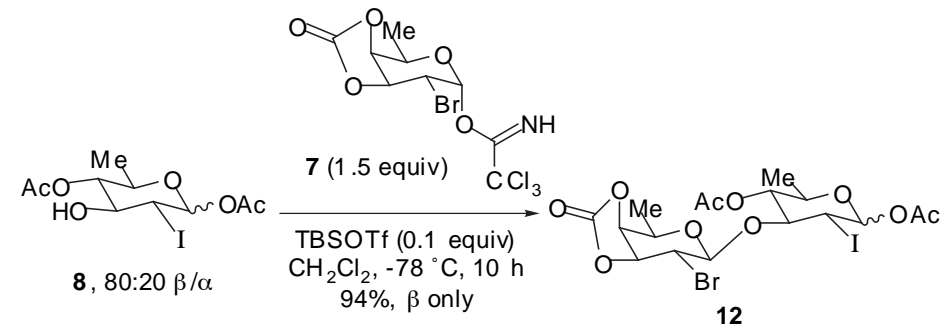

A c e t y $1 \quad 4$ - $O$-Acetyl-3- $O$-(3,4-di- $O$-(carbonyl)-2,6-dideoxy-2-bromo- $\boldsymbol{\beta}$-D-galactopyranosyl)-(1 $\rightarrow$ 3)-Dglucopyranoside ( $\beta$-12). Glucoside $8(301 \mathrm{mg}, 0.840 \mathrm{mmol})$ and donor $\mathbf{7}^{3}(498 \mathrm{mg}, 1.25 \mathrm{mmol})$ were dissolved in dry $\mathrm{CH}_{2} \mathrm{Cl}_{2}\left(4.2 \_\right.$L) under argon and the resulting solution cooled to $-78{ }^{\circ} \mathrm{C}$. To this solution was added TBSOTf $(19.0 \mu \mathrm{L}, 0.0825 \mathrm{mmol})$ and the resulting mixture was stirred for $10 \mathrm{~h}$ during which time a white precipitate formed. After this time, TLC analysis indicated all of acceptor $\mathbf{8}$ had been consumed. The reaction was quenched with neat $\mathrm{Et}_{3} \mathrm{~N}$ and warmed to $23{ }^{\circ} \mathrm{C}$. The resulting solution was diluted with EtOAc and washed with saturated $\mathrm{NaHCO}_{3}$ solution followed by saturated $\mathrm{NaCl}$. The solution was dried over anhydrous $\mathrm{Na}_{2} \mathrm{SO}_{4}$, filtered, and concentrated. The residue was purified by flash chromatography (4:1 then $2: 1$ hexanes/EtOAc) to give disaccharide 12 (469 mg, 94\%). An analytical sample of $\beta, \beta-12$ was obtaind by carrying out the reaction with $\beta-8$ (98 : $2 \beta / \alpha$, obtained through recrystallization as described above). Data for $\beta, \beta-12:[\alpha]_{\mathrm{D}}{ }^{2{ }^{\circ} \mathrm{C}}=+61.0^{\circ}(\mathrm{c}=0.2$, $\left.\mathrm{CH}_{2} \mathrm{Cl}_{2}\right) ; \mathrm{mp}=124^{\circ} \mathrm{C}(\mathrm{d}) ;{ }^{1} \mathrm{H} \mathrm{NMR}\left(400 \mathrm{MHz}, \mathrm{CDCl}_{3}\right) \delta 5.81(\mathrm{~d}, J=9.00 \mathrm{~Hz}, 1 \mathrm{H}), 5.25(\mathrm{~d}, J=6.00 \mathrm{~Hz}, 1 \mathrm{H}), 5.07$ $(\mathrm{dd}, J=7.50,6.00 \mathrm{~Hz}, 1 \mathrm{H}), 4.73(\mathrm{at}, J=9.00 \mathrm{~Hz}, 1 \mathrm{H}), 4.59(\mathrm{dd}, J=7.50,2.00 \mathrm{~Hz}, 1 \mathrm{H}), 4.16(\mathrm{dq}, J=6.50,2.00 \mathrm{~Hz}$, $1 \mathrm{H}), 3.97-4.05(\mathrm{~m}, 3 \mathrm{H}), 3.60(\mathrm{~m}, 1 \mathrm{H}), 2.16(\mathrm{~s}, 3 \mathrm{H}), 2.08(\mathrm{~s}, 3 \mathrm{H}), 1.44(\mathrm{~d}, J=7.00 \mathrm{~Hz}, 1 \mathrm{H}), 1.22(\mathrm{~d}, J=6.50 \mathrm{~Hz}$, $1 \mathrm{H}) ;{ }^{13} \mathrm{C} \mathrm{NMR}\left(100 \mathrm{MHz}, \mathrm{CDCl}_{3}\right) \delta 169.65,169.13,153.15,101.09,93.92,82.98,78.68,76.16,73.97,71.29,67.76$, 45.18, 29.27, 21.22, 20.95, 17.52, 16.67; IR (thin film) 1818, $1754 \mathrm{~cm}^{-1}$; HRMS (ES) calcd for $\mathrm{C}_{22} \mathrm{H}_{22} \mathrm{BrIO}_{10} \mathrm{Na}$ $(\mathrm{M}+\mathrm{Na})^{+} 614.9339$, found $614.9337 \mathrm{~m} / \mathrm{z}$.

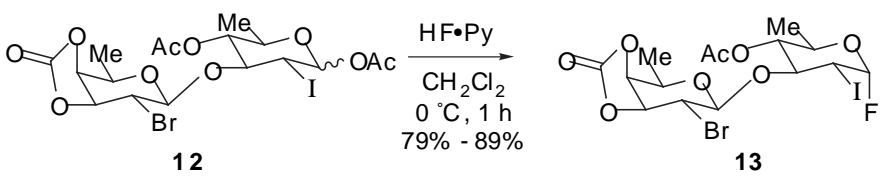

4- $O$-Acetyl-3- $O$-(3,4-di- $O$-(carbonyl)-2,6-dideoxy-2-bromo- $\beta$-D-galactopyranosyl)-(1 $\rightarrow 3)$ - $\alpha$-D-

glucopyranosyl Fluoride (13). Acetate $12(100 \mathrm{mg}, 0.169 \mathrm{mmol})$ was dissolved in dry $\mathrm{CH}_{2} \mathrm{Cl}_{2}(3.6 \mathrm{~mL})$ under nitrogen and the resulting solution was cooled to $0{ }^{\circ} \mathrm{C}$. HF•Pyridine $(65-70 \%, 270 \mu \mathrm{L})$ was added and the resulting mixture stirred for 75 min. The reaction was diluted with $\mathrm{CH}_{2} \mathrm{Cl}_{2}$ and washed with $\mathrm{NaHCO}_{3}$ solution (x 2) followed by saturated $\mathrm{NaCl}$. The solution was dried over anhydrous $\mathrm{Na}_{2} \mathrm{SO}_{4}$, filtered, and concentrated. The residue was purified by flash chromatography $\left(2: 1\right.$ hexanes/EtOAc) to give fluoride $13(82 \mathrm{mg}, 88 \%)$ as a clear oil: $[\alpha]_{\mathrm{D}}{ }^{25^{\circ} \mathrm{C}}=$ $+232^{\circ}\left(\mathrm{c}=0.3, \mathrm{CH}_{2} \mathrm{Cl}_{2}\right) ;{ }^{1} \mathrm{H}$ NMR $\left(500 \mathrm{MHz}, \mathrm{CDCl}_{3}\right) \delta 5.67(\mathrm{dd}, J=50.74,2.25 \mathrm{~Hz}, 1 \mathrm{H}), 5.19(\mathrm{~d}, J=6.00 \mathrm{~Hz}, 1 \mathrm{H})$, $5.06(\mathrm{dd}, J=5.50,7.50 \mathrm{~Hz}, 1 \mathrm{H}), 4.79(\mathrm{at}, J=9.25 \mathrm{~Hz}, 1 \mathrm{H}), 4.57$ (dd, $J=7.25,1.75 \mathrm{~Hz}, 1 \mathrm{H}), 3.98-4.16(\mathrm{~m}, 5 \mathrm{H})$, $2.08(\mathrm{~s}, 3 \mathrm{H}), 1.44(\mathrm{~d}, J=6.50 \mathrm{~Hz}, 3 \mathrm{H}), 1.22(\mathrm{~d}, J=6.00 \mathrm{~Hz}, 3 \mathrm{H}) ;{ }^{13} \mathrm{C} \mathrm{NMR}\left(125 \mathrm{MHz}, \mathrm{CDCl}_{3}\right) \delta 169.62,153.14$, $107.40(\mathrm{~d} J=224 \mathrm{~Hz}), 101.15,79.49,78.75,76.16,73.51,68.78(\mathrm{~d}, J=4.5 \mathrm{~Hz}), 67.80,45.14,25.82(\mathrm{~d}, J=29 \mathrm{~Hz})$, 21.18, 17.51, 16.63; IR (thin film) 1818, 1757, $1739 \mathrm{~cm}^{-1}$; HRMS (ES) calcd for $\mathrm{C}_{15} \mathrm{H}_{19} \mathrm{BrFIO}{ }_{8} \mathrm{Na}(\mathrm{M}+\mathrm{Na})^{+}$ 574.9190 , found $574.9189 \mathrm{~m} / \mathrm{z}$. 


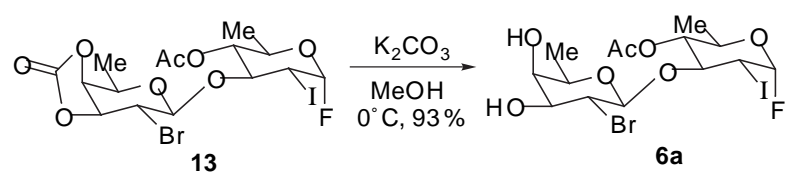

4- $O$-Acetyl-3- $O$-(2,6-dideoxy-2-bromo- $\beta$-D-galactopyranosyl)-(1 $\rightarrow 3$ )- $\alpha$-D-glucopyranosyl Fluoride (6a). Carbonate $13(170 \mathrm{mg}, 0.307 \mathrm{mmol})$ was dissolved in freshly distilled $\mathrm{MeOH}(6.5 \mathrm{~mL})$ and the resulting solution was cooled to $0{ }^{\circ} \mathrm{C}$. To this solution was added anhydrous potassium carbonate $(28 \mathrm{mg}, 0.20 \mathrm{mmol})$ and the resulting mixture was stirred vigorously. The reaction progress was followed closely by TLC analysis. Upon complete consumption of carbonate 13, the reaction mixture was added directly to a silica gel column which had been packed with hexanes. The column was washed with 1.5 column lengths of hexanes followed by $2: 1$ hexanes/EtOAc until diol 6a had been completely eluted, thereby providing the title compound $(151 \mathrm{mg}, 93 \%)$ as a white solid: $[\alpha]_{\mathrm{D}}{ }^{25^{\circ} \mathrm{C}}=$ $+221^{\circ}\left(\mathrm{c}=0.6, \mathrm{CH}_{2} \mathrm{Cl}_{2}\right) ; \mathrm{mp}=124^{\circ} \mathrm{C}(\mathrm{d}) ; \mathrm{mp}=150^{\circ} \mathrm{C}(\mathrm{d}) ;{ }^{1} \mathrm{H} \mathrm{NMR}\left(500 \mathrm{MHz}, \mathrm{CDCl}_{3}\right) \delta 5.67(\mathrm{dd}, J=2.25,50.74$ $\mathrm{Hz}, 1 \mathrm{H}), 4.97,(\mathrm{~d}, J=7.50 \mathrm{hz}, 1 \mathrm{H}), 4.75($ at $, J=9.50 \mathrm{~Hz}, 1 \mathrm{H}), 4.23(\mathrm{dd}, J=9.00,11.00 \mathrm{~Hz}, 1 \mathrm{H}), 4.12(\mathrm{dddd}, J=$ $6.00,6.00,6.00,10.00 \mathrm{~Hz}, 1 \mathrm{H}), 4.05(\mathrm{ddd}, J=2.25,11.0,28.87 \mathrm{~Hz}, 1 \mathrm{H}), 3.73-3.83(\mathrm{~m}, 3 \mathrm{H}), 3.66(\mathrm{aq}, J=6.50 \mathrm{~Hz}$, $1 \mathrm{H}), 2.81(\mathrm{bs}, 1 \mathrm{H}), 2.38(\mathrm{bs}, 1 \mathrm{H}), 2.12(\mathrm{~s}, 3 \mathrm{H}), 1.37(\mathrm{~d}, J=6.50 \mathrm{~Hz}, 3 \mathrm{H}), 1.23(\mathrm{~d}, J=6.50 \mathrm{~Hz}, 3 \mathrm{H}) ;{ }^{13} \mathrm{C} \mathrm{NMR}(100$ $\left.\mathrm{MHz}, \mathrm{CDCl}_{3}\right) \delta 170.42,107.66(\mathrm{~d}, J=224 \mathrm{~Hz}), 100.45,76.78,75.16,73.74,71.40,70.86,68.67(\mathrm{~d}, J=4.6 \mathrm{~Hz})$, $54.48,25.33$ (d, $J=29.0 \mathrm{~Hz}$ ), 21.33, 17.56, 16.40; IR (thin film) 3459, 1732(b) $\mathrm{cm}^{-1}$; HRMS (ES) calcd for $\mathrm{C}_{14} \mathrm{H}_{21} \mathrm{BrFIO}_{7} \mathrm{Na}(\mathrm{M}+\mathrm{Na})^{+} 548.9397$, found $548.9392 \mathrm{~m} / \mathrm{z}$.

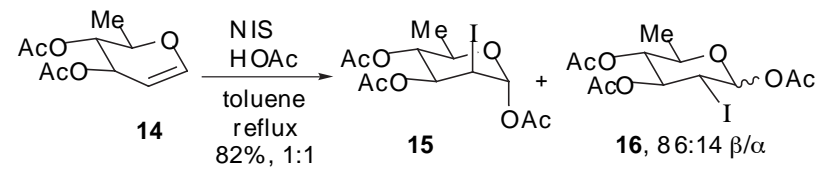

Acetyl 3,4-di- $O$-acetyl-6-deoxy-2-deoxy-2-iodo- $\alpha$-D-mannoside (15) and $\alpha, \beta$ - Acetyl 3,4-di- $O$-acetyl-6deoxy-2-deoxy-2-iodo-D-glucopyranoside $(\boldsymbol{\alpha}, \boldsymbol{\beta}-16)$. Carbohydrate derivatives 15 and 16 were prepared from 6deoxy glucal derivative $1 \mathbf{4}^{4}$ according to the haloacetoxylation procedure described for the preparation of compounds $\mathbf{1 1}$ and $\mathbf{8}$. The title compounds were isolated by flash chromatography (7:1 hexanes/EtOAc, gluco compound 16 eluted first) as yellow oils. Data for mannoside 15: $[\alpha]_{\mathrm{D}}{ }^{25^{\circ} \mathrm{C}}=+30.1^{\circ}\left(\mathrm{c}=0.8, \mathrm{CH}_{2} \mathrm{Cl}_{2}\right) ;{ }^{1} \mathrm{H} \mathrm{NMR}(500$ $\left.\mathrm{MHz}, \mathrm{CDCl}_{3}\right) \delta 6.34(\mathrm{as}, 1 \mathrm{H}), 5.20(\mathrm{~m}, 1 \mathrm{H}), 4.53(\mathrm{~m}, 2 \mathrm{H}), 4.02(\mathrm{dddd}, J=6.00,6.00,6.00,10.00 \mathrm{~Hz}, 1 \mathrm{H}), 2.16(\mathrm{~s}$, $3 \mathrm{H}), 2.11(\mathrm{~s}, 3 \mathrm{H}), 2.08(\mathrm{~s}, 3 \mathrm{H}), 1.25(\mathrm{~d}, J=6.50 \mathrm{~Hz}, 1 \mathrm{H}) ;{ }^{13} \mathrm{C} \mathrm{NMR}\left(125 \mathrm{MHz}, \mathrm{CDCl}_{3}\right) \delta 170.22,169.80,168.66$, 94.95, 72.19, 69.68, 68.94, 28.20, 21.19, 21.12, 20.95, 17.79; IR (thin film) $1749 \mathrm{~cm}^{-1}$; HRMS (ES) calcd for $\mathrm{C}_{12} \mathrm{H}_{17} \mathrm{IO}_{7} \mathrm{Na}(\mathrm{M}+\mathrm{Na})^{+} 422.9917$, found $422.9915 \mathrm{~m} / \mathrm{z}$.

Purification of glucosides $\alpha, \beta-\mathbf{1 6}$ by preparative HPLC ( $85 \%$ hexanes/EtOAc) provided an analytical sample of $\beta$-16: $[\alpha]_{\mathrm{D}}{ }^{25^{\circ} \mathrm{C}}=+110^{\circ}\left(\mathrm{c}=1.2, \mathrm{CH}_{2} \mathrm{Cl}_{2}\right) ;{ }^{1} \mathrm{H}$ NMR $\left(500 \mathrm{MHz}, \mathrm{CDCl}_{3}\right) \delta 5.85(\mathrm{~d}, J=9.50 \mathrm{~Hz}, 1 \mathrm{H}), 5.30(\mathrm{dd}, J=$ 9.00, $11.00 \mathrm{~Hz}, 1 \mathrm{H}), 4.74($ at, $J=9.25 \mathrm{~Hz}, 1 \mathrm{H}), 3.96(\mathrm{dd}, J=9.50,11.00 \mathrm{~Hz}, 1 \mathrm{H}), 3.76$ (dddd, $J=6.50,6.50,6.50$, $9.50 \mathrm{~Hz}, 1 \mathrm{H}), 2.17(\mathrm{~s}, 3 \mathrm{H}), 2.10(\mathrm{~s}, 3 \mathrm{H}), 2.04(\mathrm{~s}, 3 \mathrm{H}), 1.24(\mathrm{~d}, J=6.00 \mathrm{~Hz}, 1 \mathrm{H}) ;{ }^{13} \mathrm{C} \mathrm{NMR}\left(125 \mathrm{MHz}, \mathrm{CDCl}_{3}\right) \delta$ 169.97, 169.76, 169.89, 93.98, 75.36, 73.90, 71.36, 26.65, 20.97, 20.94, 20.88, 17.43; IR (thin film) $1755 \mathrm{~cm}^{-1}$; HRMS (ES) calcd for $\mathrm{C}_{12} \mathrm{H}_{17} \mathrm{IO}_{7} \mathrm{Na}(\mathrm{M}+\mathrm{Na})^{+} 422.9917$, found $422.9922 \mathrm{~m} / z$.
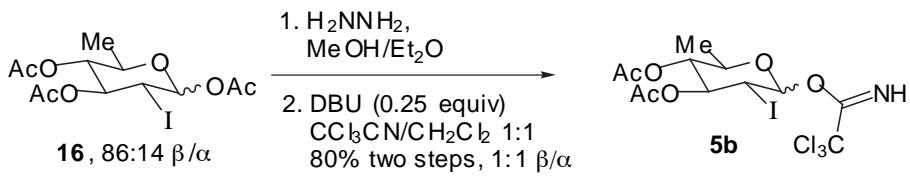

$\boldsymbol{\alpha}, \boldsymbol{\beta}$-3,4-Di- $\boldsymbol{O}$-acetyl-6-deoxy-2-deoxy-2-iodo-D-glucosyl Trichloroacetimidate $(\boldsymbol{\alpha}, \boldsymbol{\beta}-5 \mathbf{b})$. Glucoside 16 (461 $\mathrm{mg}, 1.15 \mathrm{mmol})$ was dissolved in $1: 1 \mathrm{MeOH} / \mathrm{Et}_{2} \mathrm{O}(11 \mathrm{~mL})$ and the resulting solution was cooled to $0{ }^{\circ} \mathrm{C}$. Aqueous hydrazine solution $(180 \mu \mathrm{L}, 35 \%$ wt.) was added. The progress of the reaction was monitored closely by TLC analysis. Upon complete consumption of acetate $\mathbf{1 6}(40 \mathrm{~min})$, the reaction was filtered through a pad of silica $(2: 1$ hexanes/EtOAc) and the filtrate was concentrated to yield the hemiacetal $(414 \mathrm{mg})$. The hemiacetal was dissolved in $\mathrm{CH}_{2} \mathrm{Cl}_{2}(6.5 \mathrm{~mL})$ under nitrogen and the resulting solution was cooled to $0{ }^{\circ} \mathrm{C}$. To the cold solution was added $\mathrm{Cl}_{3} \mathrm{CCN}(6.5 \mathrm{~mL})$. After several minutes, DBU $(54 \mu \mathrm{L}, 0.36 \mathrm{mmol})$ was added and the solution became orange. After $2 \mathrm{~h}$, the solution was concentrated on the rotary evaporator and the residue was chromatographed on Davisil ${ }^{\mathrm{TM}}$ silica ( $\mathrm{pH}=7.0,7: 1$ hexanes EtOAc) to give the title compound (white solid, $460 \mathrm{mg}, 80 \%$ ) as a $1: 1$ mixture of anomers. Partial data for the 1:1 mixture of imidates: ${ }^{1} \mathrm{H}$ NMR $\left(500 \mathrm{MHz}, \mathrm{C}_{6} \mathrm{D}_{6}\right) \delta 8.67(\mathrm{~s}, 1 \mathrm{H}), 8.66(\mathrm{~s}, 1 \mathrm{H}), 6.49$ $(\mathrm{d}, J=3.50 \mathrm{~Hz}, 1 \mathrm{H}), 6.04(\mathrm{~d}, J=9.50 \mathrm{~Hz}, 1 \mathrm{H}), 5.76(\mathrm{dd}, J=9.50,11.00 \mathrm{~Hz}, 1 \mathrm{H}), 5.33(\mathrm{dd}, J=9.00,11.00 \mathrm{~Hz}, 1 \mathrm{H})$, $4.86($ at, $J=9.50 \mathrm{~Hz}, 1 \mathrm{H}), 4.71($ at $, J=9.50 \mathrm{~Hz}, 1 \mathrm{H}), 4.23(\mathrm{dddd}, J=6.00,6.00,6.00,11.00 \mathrm{~Hz}, 1 \mathrm{H}), 3.82(\mathrm{dd}, J=$ 
9.50, $11.00 \mathrm{~Hz}, 1 \mathrm{H}), 3.73(\mathrm{dd}, J=3.50,11.50 \mathrm{~Hz}, 1 \mathrm{H}), 3.26(\mathrm{dddd}, J=6.00,6.00,6.00,11.00 \mathrm{~Hz}, 1 \mathrm{H}), 1.79(\mathrm{~s}, 3 \mathrm{H})$, $1.77(\mathrm{~s}, 3 \mathrm{H}), 1.66(\mathrm{~s}, 3 \mathrm{H}), 1.64(\mathrm{~s}, 3 \mathrm{H}), 1.07(\mathrm{~d}, J=6.00 \mathrm{~Hz}, 1 \mathrm{H}), 0.99(\mathrm{~d}, J=6.00 \mathrm{~Hz}, 1 \mathrm{H})$.

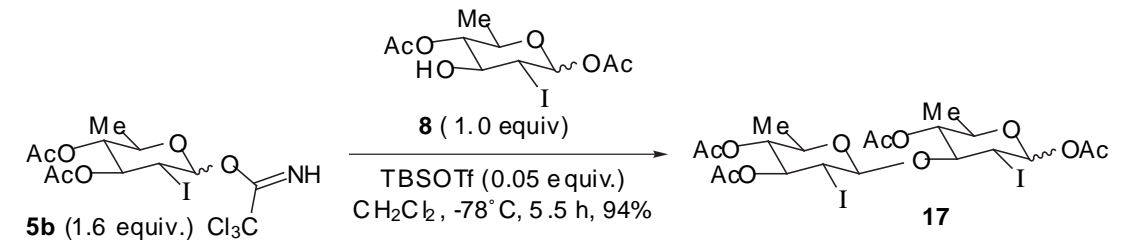

$\alpha, \beta$ - A c e $t$ y l $\quad 4$ - $O$-Acetyl-3- $O$-(3,4-di- $O$-acetyl-2,6-dideoxy-2-iodo- $\beta$-D-glucopyranosyl)-(1 $\rightarrow 3)$-Dglucopyranoside $(\boldsymbol{\beta}-\mathbf{1 7})$. Alcohol $8(145 \mathrm{mg}, 0.405 \mathrm{mmol}, 88: 12 \beta / \alpha)$ and imidate $\mathbf{5 b}(317 \mathrm{mg}, 0.631 \mathrm{mmol}) \mathrm{were}$ dissolved in dry $\mathrm{CH}_{2} \mathrm{Cl}_{2}\left(1.65 \_\mathrm{L}\right)$ under argon and the resulting solution cooled to $-78{ }^{\circ} \mathrm{C}$. To this solution was added TBSOTf $(4.7 \mu \mathrm{L}, 0.0022 \mathrm{mmol})$ and the resulting mixture was stirred for $5.5 \mathrm{~h}$. After this time, TLC analysis indicated all of acceptor 8 had been consumed and the reaction was quenched with neat $\mathrm{Et}_{3} \mathrm{~N}$ and warmed to $23{ }^{\circ} \mathrm{C}$. The resulting solution was diluted with EtOAc and washed with saturated $\mathrm{NaHCO}_{3}$ solution followed by saturated $\mathrm{NaCl}$. The solution was dried over anhydrous $\mathrm{Na}_{2} \mathrm{SO}_{4}$, filtered, and concentrated. The residue was then dissolved in dry $\mathrm{CH}_{2} \mathrm{Cl}_{2}\left(1.6 \_\mathrm{L}\right)$ under nitrogen and $\mathrm{Et}_{3} \mathrm{~N}(220 \mu \mathrm{L}, 1.58 \mathrm{mmol})$, acetic anhydride $(120 \mu \mathrm{L}, 1.26 \mathrm{mmol})$, and DMAP (2-3 crystals) and the resulting solution was stirred at $23{ }^{\circ} \mathrm{C}$ for $10.5 \mathrm{~h}$ (this step eases the subsequent purification step by converting the reaction by-products to higher $\mathrm{R}_{f}$ materials). The resulting solution was diluted with EtOAc and washed with saturated $\mathrm{NaHCO}_{3}$ solution followed by saturated $\mathrm{NaCl}$ solution. The organic phase was dried over anhydrous $\mathrm{Na}_{2} \mathrm{SO}_{4}$, filtered, and concentrated. The residue was purified by flash chromatography (2 : 1 hexanes/EtOAc) to give disaccharide $\beta-17(267 \mathrm{mg}, 94 \%)$ as a 80:20 mixture of $\beta / \alpha$ acetates: An analytical sample of $\beta, \beta-17$ was obtained by HPLC purification of this mixture using the same solvent system: $[\alpha]_{\mathrm{D}}{ }^{25^{\circ} \mathrm{C}}=$ $+145^{\circ}\left(\mathrm{c}=0.7, \mathrm{CH}_{2} \mathrm{Cl}_{2}\right) ;{ }^{1} \mathrm{H} \mathrm{NMR}\left(400 \mathrm{MHz}, \mathrm{CDCl}_{3}\right) \delta 5.82(\mathrm{~d}, J=9.60 \mathrm{~Hz}, 1 \mathrm{H}), 5.29(\mathrm{dd}, J=9.00,11.00 \mathrm{~Hz}, 1 \mathrm{~h})$, $5.24(\mathrm{~d}, J=8.80 \mathrm{~Hz}, 1 \mathrm{H}), 5.24(\mathrm{at}, J=8.80 \mathrm{~Hz}, 2 \mathrm{H}), 4.07(\mathrm{~m}, 2 \mathrm{H}), 3.75(\mathrm{dd}, J=9.00,11.40 \mathrm{~Hz}, 1 \mathrm{H}), 3.68(\mathrm{~m}, 1 \mathrm{H})$, 3.63 (dddd, $J=6.40,6.40,6.40,8.80 \mathrm{~Hz}, 1 \mathrm{H}), 2.16(\mathrm{~s}, 3 \mathrm{H}), 2.09$ (s, 3H), 2.06 (s, 3H), $2.03(\mathrm{~s}, 3 \mathrm{H}), 1.26(\mathrm{~d}, J=6.40$ $\mathrm{Hz}, 3 \mathrm{H}), 1.22(\mathrm{~d}, J=6.40 \mathrm{~Hz}, 1 \mathrm{H}) ;{ }^{13} \mathrm{C} \mathrm{NMR}\left(100 \mathrm{MHz}, \mathrm{CDCl}_{3}\right) \delta 170.06,169.99,169.75,169.18,100.52,94.08$, $80.90,75.54,74.48,74.15,71.19,70.34,28.34,27.43,21.34,21.03,20.96,20.91,17.77,17.60$; IR (thin film) 1749(b) $\mathrm{cm}^{-1}$; HRMS (ES) calcd for $\mathrm{C}_{20} \mathrm{H}_{28} \mathrm{I}_{2} \mathrm{O}_{11} \mathrm{Na}(\mathrm{M}+\mathrm{Na})^{+} 720.9619$, found $720.9622 \mathrm{~m} / \mathrm{z}$.
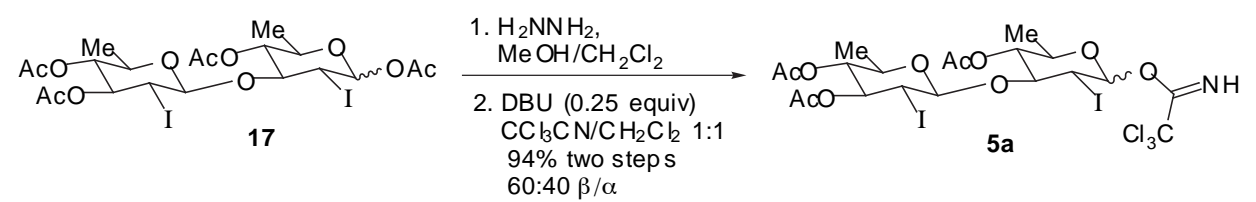

$\alpha, \beta-$ A c e t y 1

4 - $O$-Acetyl-3- $O$-(3,4-di- $O$-acetyl-2,6-dideoxy-2-iodo- $\beta$-D-glucopyranosyl)-(1 $\rightarrow$ 3)-Dglucopyranosyl Trichloroacetimidate ( $\boldsymbol{\alpha}, \boldsymbol{\beta}-\mathbf{5 a})$. $\beta$-Disaccharide $\mathbf{1 7}$ (100 $\mathrm{mg}, 0.143 \mathrm{mmol})$ was dissolved in $1: 1$ $\mathrm{MeOH} / \mathrm{CH}_{2} \mathrm{Cl}_{2}(2 \mathrm{~mL})$. Aqueous hydrazine solution $(32 \mu \mathrm{L}, 35 \%$ wt.) was added. After $2 \mathrm{~h}$, the reaction was filtered through a pad of silica (1:1 hexanes/EtOAc) and the filtrate was concentrated to yield the hemiacetal (91 mg). The hemiacetal was dissolved in $\mathrm{CH}_{2} \mathrm{Cl}_{2}(0.7 \mathrm{~mL})$ under nitrogen and the resulting solution was cooled to $0{ }^{\circ} \mathrm{C}$. To the cold solution was added $\mathrm{Cl}_{3} \mathrm{CCN}(0.7 \mathrm{~mL})$. After several minutes, DBU $(5 \mu \mathrm{L}, 0.36 \mathrm{mmol})$ was added and the solution became orange. After $1.5 \mathrm{~h}$, the solution was concentrated on the rotary evaporator and the residue was filtered through a short Davisil ${ }^{\mathrm{TM}}$ silica column $(\mathrm{pH}=7.0,2: 1$ hexanes EtOAc) to give, upon concentration of the fractions, the title compound (white solid, $108 \mathrm{mg}$, 94\% overall) as a $\sim 60: 40$ mixture of anomers: ${ }^{1} \mathrm{H}$ NMR analysis of the mixture of imidates revealed characteristic peaks which could be integrated to allow determination of the anomeric composition: ${ }^{1} \mathrm{H}$ NMR $\left(500 \mathrm{MHz}, \mathrm{C}_{6} \mathrm{D}_{6}\right) \delta 8.63(\mathrm{~s}, 1 \mathrm{H}, \beta), 8.61(\mathrm{~s}, 1 \mathrm{H}, \alpha), 6.44(\mathrm{~d}, J=3.00 \mathrm{~Hz}, 1 \mathrm{H}, \alpha)$, $6.04(\mathrm{~d}, J=9.50 \mathrm{~Hz}, 1 \mathrm{H}, \beta)$.

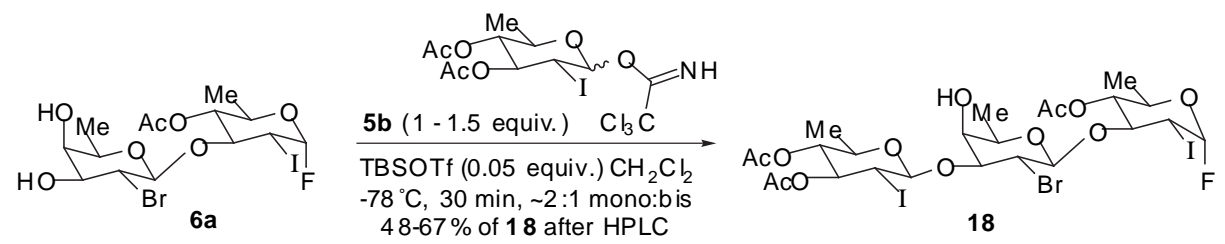

4-O-Acetyl-3-O-(3-O-(3,4-di- $O$-acetyl-6-deoxy-2-deoxy-2-iodo- $\beta$-D-glucosyl-(1 $\rightarrow 3))$-2,6-dideoxy-2-bromo$\boldsymbol{\beta}$-D-galactopyranosyl)-(1 $\rightarrow$ 3)- $\boldsymbol{\alpha}$-D-glucopyranosyl Fluoride (18). Diol 6a (22 $\mathrm{mg}, 0.042 \mathrm{mmol})$ and imidate $\mathbf{5 b}$ 
(32 mg, $0.064 \mathrm{mmol}$ ) were dissolved in dry $\mathrm{CH}_{2} \mathrm{Cl}_{2}(400 \mu \mathrm{L})$ under argon and the resulting solution cooled to -78 ${ }^{\circ} \mathrm{C}$. To this solution was added TBSOTf $(0.5 \mu \mathrm{L}, 0.0022 \mathrm{mmol})$ and the resulting mixture was stirred for $5.5 \mathrm{~h}$. The reaction was quenched with neat $\mathrm{Et}_{3} \mathrm{~N}$ and warmed to $23{ }^{\circ} \mathrm{C}$. The resulting solution was diluted with EtOAc and washed with saturated $\mathrm{NaHCO}_{3}$ solution followed by saturated $\mathrm{NaCl}$. The solution was dried over anhydrous $\mathrm{Na}_{2} \mathrm{SO}_{4}$, filtered, and concentrated. The residue was purified by flash chromatography $(4: 1$ then $2: 1$ hexanes/EtOAc) to give $35 \mathrm{mg}$ of aclear oil ( 2:1 mixture of mono:bis glycosidation products). Purification of this material by preparative HPLC (66\% hexanes/EtOAc) provided the title compound ( $24 \mathrm{mg}, 67 \%$ ) as a clear oil: $[\alpha]_{\mathrm{D}}{ }^{25^{\circ} \mathrm{C}}=+63.2^{\circ}\left(\mathrm{c}=2.4, \mathrm{CH}_{2} \mathrm{Cl}_{2}\right) ; \quad{ }^{1} \mathrm{H} \mathrm{NMR}\left(500 \mathrm{MHz}, \mathrm{CDCl}_{3}\right) \delta 5.67(\mathrm{dd}, J=2.25,50.74 \mathrm{~Hz}, 1 \mathrm{H}), 5.26(\mathrm{dd}, J=$ $9.00,11.00 \mathrm{~Hz}, 1 \mathrm{H}), 4.99(\mathrm{~d}, J=8.00 \mathrm{~Hz}, 1 \mathrm{H}), 4.93(\mathrm{~d}, J=9.00 \mathrm{~Hz}, 1 \mathrm{H}), 4.75($ at, $J=11.00 \mathrm{~Hz}, 1 \mathrm{H}), 4.72($ at, $J=$ $10.50 \mathrm{~Hz}, 1 \mathrm{H}), 4.26(\mathrm{dd}, J=9.00,11.00 \mathrm{~Hz}, 1 \mathrm{H}), 4.12(\mathrm{~m}, 1 \mathrm{H}), 4.06(\mathrm{dd}, J=2.00,11.00,29.00 \mathrm{~Hz}, 1 \mathrm{H}), 3.82-3.91$ $(\mathrm{m}, 3 \mathrm{H}), 3.72(\mathrm{~m}, 1 \mathrm{H}), 3.60-3.66(\mathrm{~m}, 2 \mathrm{H}), 2.44(\mathrm{~m}, 1 \mathrm{H}), 2.13(\mathrm{~s}, 3 \mathrm{H}), 2.10(\mathrm{~s}, 3 \mathrm{H}), 2.03(\mathrm{~s}, 3 \mathrm{H}), 1.37(\mathrm{~d}, J=6.50$ $\mathrm{Hz}, 1 \mathrm{H}), 1.23(\mathrm{~d}, J=6.50 \mathrm{~Hz}, 6 \mathrm{H}) ;{ }^{13} \mathrm{C} \mathrm{NMR}\left(100 \mathrm{MHz}, \mathrm{CDCl}_{3}\right) \delta 170.37,169.94,107.73(\mathrm{~d}, J=224 \mathrm{~Hz}), 103.08$, $100.57,83.52,76.24,75.42,74.07,73.66,71.64,70.59,70.23,68.76(\mathrm{~d}, J=4.7 \mathrm{~Hz}), 50.09,27.05,25.39(\mathrm{~d}, J=29.0$ $\mathrm{Hz}$ ), 21.24, 21.02, 20.89, 17.64, 17.55, 16.46; IR (thin film) 3540, $1750 \mathrm{~cm}^{-1}$; HRMS (ES) calcd for $\mathrm{C}_{24} \mathrm{H}_{34} \mathrm{BrFI}_{2} \mathrm{O}_{12} \mathrm{Na}(\mathrm{M}+\mathrm{Na})^{+} 888.9205$, found $888.9207 \mathrm{~m} / \mathrm{z}$.
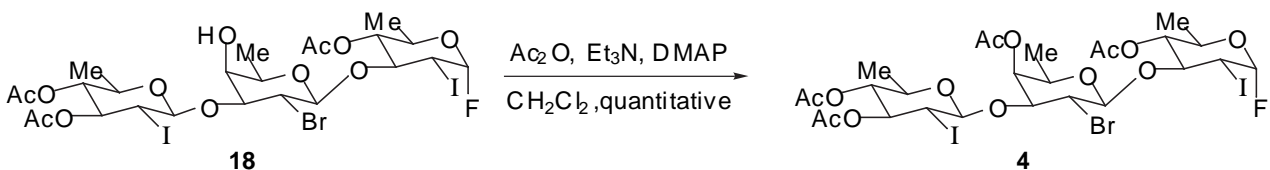

4-O-Acetyl-3- $O$-(4- $O$-acetyl-3- $O$-(3,4-di- $O$-acetyl-6-deoxy-2-deoxy-2-iodo- $\beta$-D-glucosyl-(1 $\rightarrow 3$ ))-2,6dideoxy-2-bromo- $\boldsymbol{\beta}$-D-galactopyranosyl)-(1 $\rightarrow \mathbf{3}$ )- $\boldsymbol{\alpha}$-D-glucopyranosyl Fluoride (4). Diol 18 (33 mg, $0.038 \mathrm{mmol})$ was dissolved in dry $\mathrm{CH}_{2} \mathrm{Cl}_{2}\left(1.0 \_\mathrm{L}\right)$ under a nitrogen atmosphere and DMAP $(10 \mathrm{mg}, 0.082 \mathrm{mmol}), \mathrm{Et}_{3} \mathrm{~N}(28 \mu \mathrm{L}$, $0.20 \mathrm{mmol})$, and $\mathrm{Ac}_{2} \mathrm{O}(14 \mu \mathrm{L}, 0.15 \mathrm{mmol})$ were added successively. The resulting mixture was stirred for $24 \mathrm{~h}$ and then diluted with EtOAc and washed with saturated $\mathrm{NaHCO}_{3}$ solution followed by saturated $\mathrm{NaCl}$. The solution was dried over anhydrous $\mathrm{Na}_{2} \mathrm{SO}_{4}$, filtered, and concentrated. The residue was purified by flash chromatography (2:1 hexanes/EtOAc) to give the title compound (35 mg, quantitative) as glass: $[\alpha]_{\mathrm{D}}{ }^{25^{\circ} \mathrm{C}}=139^{\circ}\left(\mathrm{c}=0.3, \mathrm{CH}_{2} \mathrm{Cl}_{2}\right) ;{ }^{1} \mathrm{H}$ NMR $\left(500 \mathrm{MHz}, \mathrm{CDCl}_{3}\right) \delta 5.67(\mathrm{dd}, J=2.00,50.49 \mathrm{~Hz}, 1 \mathrm{H}), 5.23(\mathrm{dd}, J=9.00,11.00 \mathrm{~Hz}, 1 \mathrm{H}), 5.18(\mathrm{dd}, J=3.25$, $0.75 \mathrm{~Hz}, 1 \mathrm{H}), 5.04(\mathrm{~d}, J=8.00 \mathrm{~Hz}, 1 \mathrm{H}), 4.95(\mathrm{~d}, J=9.00 \mathrm{~Hz}, 1 \mathrm{H}), 4.77($ at, $J=10.00 \mathrm{~Hz}, 1 \mathrm{H}), 4.70($ at, $J=9.25$ $\mathrm{Hz}, 1 \mathrm{H}), 4.25(\mathrm{dd}, J=8.75,10.75,1 \mathrm{H}), 4.11(\mathrm{~m}, 1 \mathrm{H}), 4.07(\mathrm{ddd}, J=2.25,11.00,28.49 \mathrm{~Hz}, 1 \mathrm{H}), 3.96(\mathrm{dd}, J=3.25$, $10.75 \mathrm{~Hz}, 1 \mathrm{H}), 3.91(\mathrm{dd}, J=8.25,10.75 \mathrm{~Hz}, 1 \mathrm{H}), 3.75(\mathrm{dd}, J=9.00,11.00 \mathrm{~Hz}, 1 \mathrm{H}), 3.58$ (dddd, $J=6.00,6.00,6.00$, $9.50 \mathrm{~Hz}, 1 \mathrm{H}), 2.12(\mathrm{~s}, 3 \mathrm{H}), 2.09(\mathrm{~s}, 3 \mathrm{H}), 2.08(\mathrm{~s}, 3 \mathrm{H}), 2.02(\mathrm{~s}, 3 \mathrm{H}), 1.23(\mathrm{~d}, J=6.50 \mathrm{~Hz}, 3 \mathrm{H}), 1.23(\mathrm{~d}, J=6.50 \mathrm{~Hz}$, $3 \mathrm{H}), 1.20(\mathrm{~d}, J=6.00 \mathrm{~Hz}, 3 \mathrm{H}) ;{ }^{13} \mathrm{C} \mathrm{NMR}\left(100 \mathrm{MHz}, \mathrm{CDCl}_{3}\right) \delta 170.18,169.96,169.94,169.84,107.72(\mathrm{~d}, J=224$ $\mathrm{Hz}), 102.56,100.59,79.37,76.44,75.50,74.23,73.73,72.96,70.49,69.83,68.73,50.41,31.80,27.46,25.11(\mathrm{~d}, J=$ $29.0 \mathrm{~Hz}$ ), 22.87, 21.11, 21.02, 21.00, 20.90, 17.53, 17.42, 16.56, 14.34; IR (thin film) $1749 \mathrm{~cm}^{-1}$; HRMS (ES) calcd for $\mathrm{C}_{26} \mathrm{H}_{36} \mathrm{BrFI}_{2} \mathrm{O}_{13} \mathrm{Na}(\mathrm{M}+\mathrm{Na})^{+} 930.9311$, found $930.9333 \mathrm{~m} / \mathrm{z}$.

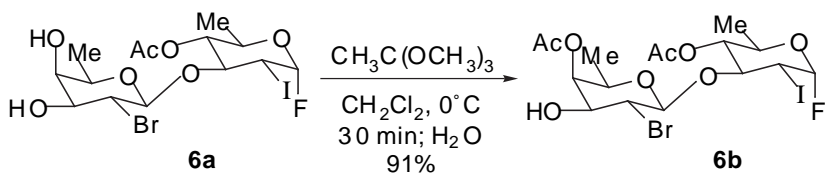

4- $O$-Acetyl-3- $O$-(4- $O$-acetyl-2,6-dideoxy-2-bromo- $\beta$-D-galactopyranosyl)-(1 $\rightarrow 3)$ - $\alpha$-D-glucopyranosyl

Fluoride (6b). Diol 6a $(86 \mathrm{mg}, 0.16 \mathrm{mmol})$ was dissolved in dry $\mathrm{CH}_{2} \mathrm{Cl}_{2}\left(1.6 \_\mathrm{L}\right)$ under nitrogen and cooled to $0^{\circ} \mathrm{C}$. Trimethylorthoacetate $(32 \mu \mathrm{L}, 0.250 \mathrm{mmol})$ was added to the reaction by syringe followed by the addition of $\mathrm{TsOH} \cdot \mathrm{H}_{2} \mathrm{O}(4 \mathrm{mg}, 0.02 \mathrm{mmol}){ }^{5}$ After $30 \mathrm{~min}$, water $(50 \mu \mathrm{L})$ was added and the reaction was stirred vigorously for $15 \mathrm{~min}$. The reaction was quenched with saturated sodium bicarbonate solution and then diluted with EtOAc. The aqueous layer was discarded and the organics washed with brine, dried over anhydrous $\mathrm{Na}_{2} \mathrm{SO}_{4}$, filtered, and concentrated. The residue was purified by flash chromatography $(2: 1$ hexanes/EtOAc) to give the title compound $(85 \mathrm{mg}, 91 \%)$ as a white solid: $[\alpha]_{\mathrm{D}}{ }^{25^{\circ} \mathrm{C}}=+150^{\circ}\left(\mathrm{c}=0.2, \mathrm{CH}_{2} \mathrm{Cl}_{2}\right) ; \mathrm{mp}=150^{\circ} \mathrm{C}(\mathrm{d}) ;{ }^{1} \mathrm{H} \mathrm{NMR}\left(400 \mathrm{MHz}, \mathrm{CDCl}_{3}\right) \delta$ $5.66(\mathrm{dd}, J=2.20,50.80 \mathrm{~Hz}, 1 \mathrm{H}), 5.15(\mathrm{ad}, J=3.20 \mathrm{~Hz}, 1 \mathrm{H}), 5.01(\mathrm{~d}, J=8.00 \mathrm{hz}, 1 \mathrm{H}), 4.75($ at, $J=9.60 \mathrm{~Hz}, 1 \mathrm{H})$, $4.21(\mathrm{dd}, J=9.00,10.60 \mathrm{~Hz}, 1 \mathrm{H}), 3.99-4.13(\mathrm{~m}, 2 \mathrm{H}), 3.90(\mathrm{dt}, J=3.70,10.67 \mathrm{~Hz}, 1 \mathrm{H}), 3.73-3.80(\mathrm{~m}, 2 \mathrm{H}), 2.69(\mathrm{~d}, J$ $=4.00 \mathrm{~Hz}, 1 \mathrm{H}), 2.14(\mathrm{~s}, 3 \mathrm{H}), 2.09(\mathrm{~s}, 3 \mathrm{H}), 1.21(\mathrm{~d}, J=6.40 \mathrm{~Hz}, 6 \mathrm{H}) ;{ }^{13} \mathrm{C} \mathrm{NMR}\left(100 \mathrm{MHz}, \mathrm{CDCl}_{3}\right) \delta 171.06$, $169.90,107.63(\mathrm{~d}, J=224 \mathrm{~Hz}), 100.44,76.59,73.71,73.54,72.30,69.74,68.65,53.87,25.05(\mathrm{~d}, J=29.8 \mathrm{~Hz})$, 21.10, 20.87, 17.50, 16.42; IR (thin film) $3479,1742 \mathrm{~cm}^{-1}$; HRMS (ES) calcd for $\mathrm{C}_{16} \mathrm{H}_{23} \mathrm{BrFIO}_{8} \mathrm{Na}(\mathrm{M}+\mathrm{Na})^{+}$ 590.9503 , found $590.9517 \mathrm{~m} / \mathrm{z}$. 


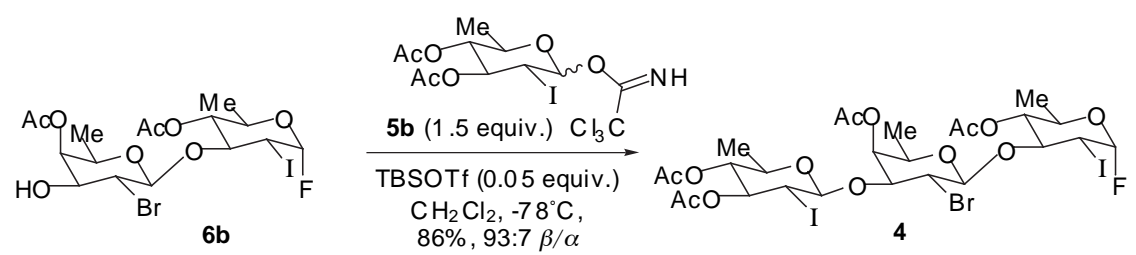

Synthesis of Trisaacharide 4 from Acetate $\mathbf{6 b}$. Acetate $\mathbf{6 b}(26 \mathrm{mg}, 0.046 \mathrm{mmol})$ and imidate $\mathbf{5 b}(38 \mathrm{mg}, 0.076$ mmol) were dissolved in dry $\mathrm{CH}_{2} \mathrm{Cl}_{2}(250 \mu \mathrm{L})$ under argon and the resulting solution cooled to $-78{ }^{\circ} \mathrm{C}$. To this solution was added TBSOTf $(0.5 \mu \mathrm{L}, 0.0022 \mathrm{mmol})$ and the resulting mixture was stirred for $1 \mathrm{~h}$. The reaction was quenched with neat $\mathrm{Et}_{3} \mathrm{~N}$ and warmed to $23{ }^{\circ} \mathrm{C}$. The resulting solution was diluted with EtOAc and washed with saturated $\mathrm{NaHCO}_{3}$ solution followed by saturated $\mathrm{NaCl}$ solution. The organic phase was dried over anhydrous $\mathrm{Na}_{2} \mathrm{SO}_{4}$, filtered, and concentrated. The residue was purified by flash chromatography ( $2: 1$ hexanes/EtOAc) to give trisaccharide $4(36 \mathrm{mg}, 86 \%)$ as a glass.

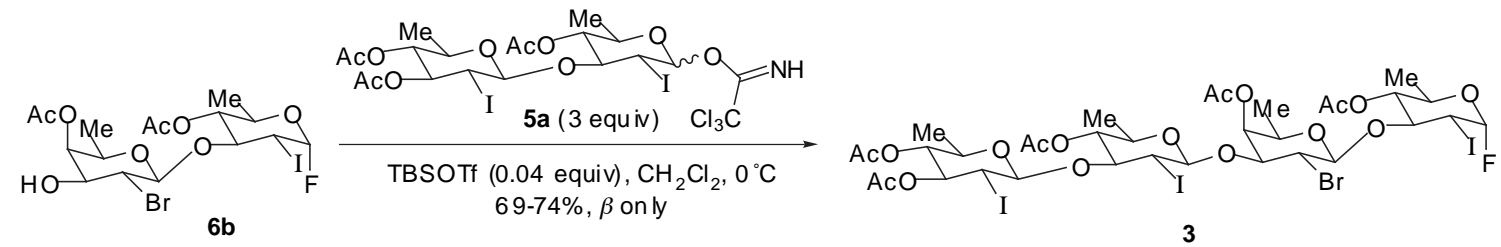

4-O-Acetyl-3- $O$-(4- $O$-acetyl-3- $O$-(3,4-di- $O$-acetyl-6-deoxy-2-deoxy-2-iodo- $\beta$-D-glucosyl-(1 $\rightarrow 3)-4-O$ acetyl-6-deoxy-2-deoxy-2-iodo- $\beta$-D-glucosyl-(1 $\rightarrow 3$ ))-2,6-dideoxy-2-bromo- $\beta$-D-galactopyranosyl)-(1 $\rightarrow 3$ )- $\alpha$-Dglucopyranosyl Fluoride (3). Acetate $\mathbf{6 b}(18 \mathrm{mg}, 0.032 \mathrm{mmol})$ and imidate $5 \mathbf{a}(83 \mathrm{mg}, 0.10 \mathrm{mmol})$ were dissolved in dry $\mathrm{CH}_{2} \mathrm{Cl}_{2}(200 \mu \mathrm{L})$ under argon and the resulting solution cooled to $0{ }^{\circ} \mathrm{C}$. To this solution was added TBSOTf $(0.3 \mu \mathrm{L}, 0.0013 \mathrm{mmol})$ and the resulting mixture was stirred for $75 \mathrm{~min}$. The reaction was quenched with neat $\mathrm{Et}_{3} \mathrm{~N}$ and warmed to $23{ }^{\circ} \mathrm{C}$. The resulting solution was diluted with EtOAc and washed with saturated $\mathrm{NaHCO}_{3}$ solution followed by saturated $\mathrm{NaCl}$ solution. The organic phase was dried over anhydrous $\mathrm{Na}_{2} \mathrm{SO}_{4}$, filtered, and concentrated. The residue was purified by flash chromatography $(7: 1$ then $4: 1$ hexanes/EtOAc) to give tetrasaccharide $3(28 \mathrm{mg}, 86 \%)$ as a white solid: $[\alpha]_{\mathrm{D}}{ }^{25^{\circ} \mathrm{C}}=+230^{\circ}\left(\mathrm{c}=0.1, \mathrm{CH}_{2} \mathrm{Cl}_{2}\right) ; \mathrm{mp}=147^{\circ} \mathrm{C}(\mathrm{d}) ;{ }^{1} \mathrm{H} \mathrm{NMR}(500$ $\left.\mathrm{MHz}, \mathrm{CDCl}_{3}\right) \delta 5.67(\mathrm{dd}, J=2.25,50.74 \mathrm{~Hz}, 1 \mathrm{H}), 5.28(\mathrm{dd}, J=9.00,11.00 \mathrm{~Hz}, 1 \mathrm{H}), 5.28(\mathrm{~d}, J=9.00 \mathrm{~Hz}, 1 \mathrm{H}), 5.17$ $(\mathrm{dd}, J=0.75,3.25 \mathrm{~Hz}, 1 \mathrm{H}), 5.04(\mathrm{~d}, J=8.00 \mathrm{~Hz}, 1 \mathrm{H}), 4.91(\mathrm{~d}, J=9.00 \mathrm{~Hz}, 1 \mathrm{H}), 4.77($ at $, J=9.50 \mathrm{hz}, 1 \mathrm{H}), 4.64$ (at, $J=9.25 \mathrm{~Hz}, 1 \mathrm{H}), 4.63$ (at, $J=9.25 \mathrm{~Hz}, 1 \mathrm{H}), 4.91(\mathrm{dd}, J=9.00,11.00 \mathrm{~Hz}, 1 \mathrm{H}), 4.01-4.14(\mathrm{~m}, 3 \mathrm{H}), 3.90-3.96(\mathrm{~m}$, 2H), $3.86(\mathrm{dd}, J=9.00,10.50 \mathrm{~Hz}, 1 \mathrm{H}), 3.73(\mathrm{dd}, J=9.00,11.50 \mathrm{~Hz}, 1 \mathrm{H}), 3.72(\mathrm{dd}, J=1.00,11.50 \mathrm{~Hz}, 1 \mathrm{H}), 3.60$ (dddd, $J=6.00,6.00,6.00,10.0 \mathrm{~Hz}, 1 \mathrm{H}$ ), 3.50 (dddd, $J=6.00,6.00,6.00,10.00 \mathrm{~Hz}, 1 \mathrm{H}), 2.12$ (s, 3H), 2.09 (s, 3H), $2.08(\mathrm{~s}, 3 \mathrm{H}), 2.04(\mathrm{~s}, 3 \mathrm{H}), 2.02(\mathrm{~s}, 3 \mathrm{H}), 1.24(\mathrm{~d}, J=6.50 \mathrm{~Hz}, 3 \mathrm{H}), 1.23(\mathrm{~d}, J=6.50 \mathrm{~Hz}, 3 \mathrm{H}), 1.21(\mathrm{~d}, J=6.00 \mathrm{~Hz}$, $3 \mathrm{H}), 1.20(\mathrm{~d}, J=6.00 \mathrm{~Hz}, 3 \mathrm{H}) ;{ }^{13} \mathrm{C} \mathrm{NMR}\left(100 \mathrm{MHz}, \mathrm{CDCl}_{3}\right) \delta 170.16,170.06,169.99,169.87,169.71,107.70(\mathrm{~d}, J$ $=224 \mathrm{~Hz}), 102.56,100.61,100.50,80.80,79.23,76.41,75.61,74.57,73.73,72.97,70.29,69.90,68.73(\mathrm{~d}, J=3.1$ $\mathrm{Hz}$ ), 50.65, 28.98, 27.34, $25.19(\mathrm{~d}, J=29.0 \mathrm{~Hz}), 21.39,21.12,21.02(\mathrm{~b}), 20.93,17.76,17.61,17.53$, 16.56; IR (thin film) $1750 \mathrm{~cm}^{-1}$; HRMS (ES) calcd for $\mathrm{C}_{34} \mathrm{H}_{47} \mathrm{BrFI}_{3} \mathrm{O}_{17} \mathrm{Na}(\mathrm{M}+\mathrm{Na})^{+} 1228.9013$, found $1228.9036 \mathrm{~m} / \mathrm{z}$.

(1) Pangborn, A. B.; Giardello, M. A.; Grubbs, R. H.; Rosen, R. K.; Timmers, F. J. Organometallics 1996, 15, 1518.

(2) Roush, W. R.; Sebesta, D. P.; Bennett, C. E. Tetrahedron 1997, 53, 8825-8836.

(3) Durham, T. B.; Roush, W. R. Org. Lett. 2003, 5, see previous letter in this issue.

(4) Tulshian, D. B.; Fraser-Reid, B. J. Am. Chem. Soc. 1981, 103, 474-475.

(5) Oikawa, M.; Wada, A.; Okazaki, F.; Kusumoto, S. J. Org. Chem. 1996, 61, 4469-4471. 